\title{
Impact of oral contraceptives and breastfeeding on disease activity in a sample of Egyptian rheumatoid arthritis patients
}

\author{
Sahar A. Elsayed*i(D, Doris M. Henry and Esam M. Abu Elfadl
}

\begin{abstract}
Background: Women are more affected by rheumatoid arthritis (RA) than men and the incidence of RA in women increases around the age of menopause indicating that hormonal factors may have a role in disease pathogenesis and progression. Despite several studies on the role of sex hormones and oral contraceptives (OCs) in RA patients, the effect of combined oral contraceptives on RA disease activity is still controversial, and since few studies have been performed in Egypt on this issue so we aimed to study the effect of OCs, breastfeeding, and prolactin on disease activity and their relation to the titer of autoantibodies in female Egyptian RA patients.

Results: One hundred twenty married female RA patients were classified into three groups based on the OCs use $(n=40$ each); current, past and non-users and according to lactation into three groups; lactating using OCs $(n=22)$, lactating not using OCs $(n=30)$, and non-lactating $(n=68)$. Our patients were using combined estrogen-progestin oral contraceptives. Clinical manifestations, disease activity, and laboratory findings were determined. The clinical manifestations including arthritis, and morning stiffness were significantly reduced in current users in comparison to past and non-users. Mean DAS28 was reduced significantly $(p<0.05)$ in current and past users of OCs. Also, using OCs during lactation reduced the clinical manifestations significantly. Lactating women who were not using OCs had significantly high DAS28 and ESR $(p<0.05)$ in comparison to lactating using OCs and non-lactating. On the other hand, using OCs reduced serum prolactin significantly. Lactating women not using OCs had significantly high serum prolactin $(p<0.05)$ and $(p<0.001)$ in comparison to lactating using OCs and non-lactating respectively which was correlated with DAS28 score.
\end{abstract}

Conclusion: Oral contraceptives may be considered in RA female patients not only as a suitable birth control method but also it has a controlling effect on disease activity even during lactation.

Message: Oral contraceptives ameliorate disease activity in RA patients

Keywords: Oral contraceptives, Breastfeeding, Rheumatoid arthritis, Disease activity

\section{Background}

Rheumatoid arthritis (RA) is an autoimmune disease that primarily targets joints, and also body systems and internal organs may be affected [1]. Its etiology is unknown but genetic, environmental, and hormonal factors may be

\footnotetext{
* Correspondence: saharomar2000@yahoo.co.uk

Department of Rheumatology and rehabilitation, Faculty of Medicine, Sohag University, Sohag 82524, Egypt
}

\section{Springer Open}

(c) The Author(s). 2020 Open Access This article is licensed under a Creative Commons Attribution 4.0 International License, which permits use, sharing, adaptation, distribution and reproduction in any medium or format, as long as you give appropriate credit to the original author(s) and the source, provide a link to the Creative Commons licence, and indicate if changes were made. The images or other third party material in this article are included in the article's Creative Commons licence, unless indicated otherwise in a credit line to the material. If material is not included in the article's Creative Commons licence and your intended use is not permitted by statutory regulation or exceeds the permitted use, you will need to obtain permission directly from the copyright holder. To view a copy of this licence, visit http://creativecommons.org/licenses/by/4.0/.

involved [2]. The fact that women are more affected by RA than men (3:1) may suggest a possible role for sex hormones [3]. The peak incidence of RA in women occurs mainly in the fifth decade of life around the menopausal age [4], but it may also occur at a younger age, and the disease is more severe and progressive in women when compared to men [5]. RA has an impact on women's health, increasing the risk of cardiovascular diseases, osteoporosis, malignancies, and other comorbidities leading to increased 
mortality [6-8]. Initial studies focused on comparing the prevalence of RA in both genders, while recent studies give more attention to sex hormones and reproductive factors but still their role in RA is unclear [9].

The female hormonal milieu is altered by multiple factors like breastfeeding, pregnancy, postpartum, menopause, and the use of oral contraceptives (OCs) $[10,11]$. It is reported that the incidence of RA in women increases around the age of menopause indicating that hormonal factors may have a role in disease pathogenesis and progression [12]. Hormonal theory may be reinforced by the fact that the incidence of RA decreases during pregnancy and RA women who get pregnant usually have disease remission [13]. However, the disease exacerbates after delivery due to the rapid decline in both estrogens and cortisol, and the significant increase in prolactin [14]. The different distribution and character of the autoimmune diseases between genders may be related to sex hormones. Prolactin is known to have an immune-stimulatory role and has a role in the pathogenesis of many autoimmune diseases. It is also related to disease relapse which occurs during pregnancy in SLE and lactation in RA patients [15].

Breastfeeding is considered as a risk factor for more aggressive inflammatory polyarthritis in women who have genetic susceptibility due to the association between HLA-DRB1 alleles and the gene of prolactin [16]. Prolactin is a hormone which has a cytokine-like action and is considered as an immune-stimulatory agent that is involved in synovial hyperplasia and inflammation [17, 18], it is secreted by the pituitary gland in addition to macrophages and lymphocytes located in the synovial tissue of inflammatory arthritis patients [19]. Prolactin has a role in the severity of the disease and enhances RA joint damage [20]. Clinical trials revealed significantly high serum prolactin in RA patients suggesting that prolactin may have a significant role in disease activity and also in the disease development process [21].

Using OCs is associated with a milder form of the disease and reduced disability [22]. Chen et al. reported that the use of OCs does not decrease the incidence of RA but may reduce disease severity and progression [23]. Despite several studies on the role of sex hormones and OCs in RA patients, their effect is still controversial especially it may be affected by race and environmental circumstances, and since as far as we know, no similar studies have been performed in Egypt yet so we aimed to study the effect of both OCs and breastfeeding on disease activity and their relation to the titer of autoantibodies in RA Egyptian female patients.

\section{Methods}

In this study, the data for 120 married female RA patients were collected from the department of rheumatology, from September 2019 to March 2020. Our patients were diagnosed according to the 2010 ACR revised criteria for RA [24]. Patients with concomitant systemic diseases and other autoimmune diseases including SLE, scleroderma, mixed connective tissue disease, Sjogren's syndrome, polymyositis, pregnancy, patients receiving hormonal therapy, and patients with a history of thromboembolic events were excluded. Written informed consent was taken from all patients, and ethical approval was obtained from the ethical committee of faculty of medicine.

Patients were classified into three groups based on OCs use; patients currently using OCs (current users), patients who stopped using OCs for at least 1 year (past users), patients who never used OCs (non-users) with 40 patients in each group. Our selected patients were using combined estrogen-progestin oral contraceptives for a minimum duration of 6 months. Lactating patients started using OCs 6 weeks postpartum. According to lactation, our patients were classified into three groups; the first group included twenty-two lactating patients using OCs (Lact. using OCs), the second group, included 30 lactating patients not using OCs (Lact. not using OCs), the third group, included 68 non-lactating patients (non-lactating).

Our patients were evaluated for demographic data (age $>18$ years, disease duration 2-8 years), current medications and clinical manifestations including morning stiffness, arthritis, and extra-articular manifestations including; rheumatoid nodules, Raynaud's phenomena, and carpal tunnel syndrome, in addition to the laboratory findings including, erythrocyte sedimentation rate, $\mathrm{CBC}, \mathrm{RF}$, and ACPA were determined. RF was determined using RF-latex slide agglutination kit (SPINREAC $\mathrm{T}$, Spain) according to the manufacturer's protocol. ACPA was quantified using ELISA technique for human IgG autoantibodies to cyclic citrullinated peptides in the serum according to the manufacturer company protocol (Demeditec Diagnostics GmbH, Germany). Serum prolactin was determined using the Quantikine ${ }^{\bullet}$ ELISA kit according to the enclosed protocol provided by $R \& D$ Systems, Inc.

Disease activity was assessed using DAS28. Visual ana$\log$ scale (VAS) was used by patients through scoring their pain ranging from 0 (no pain) to 100 (the worst pain imaginable). Tender and swollen joints were counted and ESR was recorded. Regarding the DAS28 score, disease activity was classified as remission $(<2.6)$, low disease activity (2.6-3.2), moderate disease activity (3.2-5.1), and high disease activity (> 5.1).

\section{Statistical analysis}

SPSS, version 20 (IBM Corp., Armonk, USA) was used for the data analysis. We used the Kolmogorov-Smirnov 
test to check the normal distribution of data. Data and results were expressed in mean with standard deviation or SEM in normally distributed data. Mann-Whitney $U$ test was used to compare two groups for not normally distributed data and independent $t$ test for normally distributed data.

\section{Results}

Our study included 120 married female RA patients. Their age range was 19-40 years and disease duration was 2-8 years. Our patients were classified according to OCs use and lactation as shown in the flow diagram (Fig. 1). The current lines of treatment for our patients are shown in Table 1, where $15.83 \%$ of the patients were on methotrexate (MTX), 13.33\% were on MTX + hydroxychloroquine, $6.66 \%$ were on MTX + sulphasalazine + hydroxychloroquine, $10.83 \%$ were on leflunomide, $10 \%$ were on MTX + leflunomide, $29.19 \%$ were on corticosteroids + hydroxychloroquine, and $14.16 \%$ were on corticosteroids + sulphasalazine (Table 1). None of our selected patients were using biological therapy as their count was extremely low so they are excluded at the start of the study. The lactating patients were using only two lines of treatment, corticosteroids + hydroxychloroquine and corticosteroids + sulphasalazine, which make it easy to compare, while in other groups different lines of therapy were used which make it difficult to compare.

\section{Clinical manifestations for current, past, and non-oral contraceptives users}

As shown in Table 2, using oral contraceptives lowered the mean rank of morning stiffness between the studied groups; women currently using OCs had significantly lower mean rank of morning stiffness in comparison to past users and non-users $(p<0.05)$ and $(p<0.001)$ respectively. Also, the past users of OCs had significantly $(p<0.05)$ lower mean rank of morning stiffness in comparison to non-users. On the other hand, there was no significant difference between the current and past users of OCs concerning arthritis; however, past users had a significantly lower $(p<0.05)$ mean rank of arthritis in comparison to non-users, and a highly significant $(p<0.001)$ lower mean rank of arthritis for current users in comparison to non-users. Meanwhile, using OCs had no significant effect on the extra-articular manifestations between the studied groups.

Clinical manifestations in lactating women using and not using oral contraceptives, and non-lactating

Using oral contraceptives during lactation significantly reduced $(p<0.05)$ the mean rank of morning stiffness in lactating using $\mathrm{OCs}$ in comparison to nonlactating. However, no significant difference was found between lactating using and not using OCs also, between lactating not using $\mathrm{OCs}$ and nonlactating. On the other hand, using OCs during lactation significantly reduced the mean rank of arthritis $(p<0.001)$ and $(p<0.01)$ in comparison to those who are lactating not using OCs and non-lactating respectively. Meanwhile, using OCs during lactation had no significant effect on the extra-articular manifestations in all studied groups as shown in Table 3.

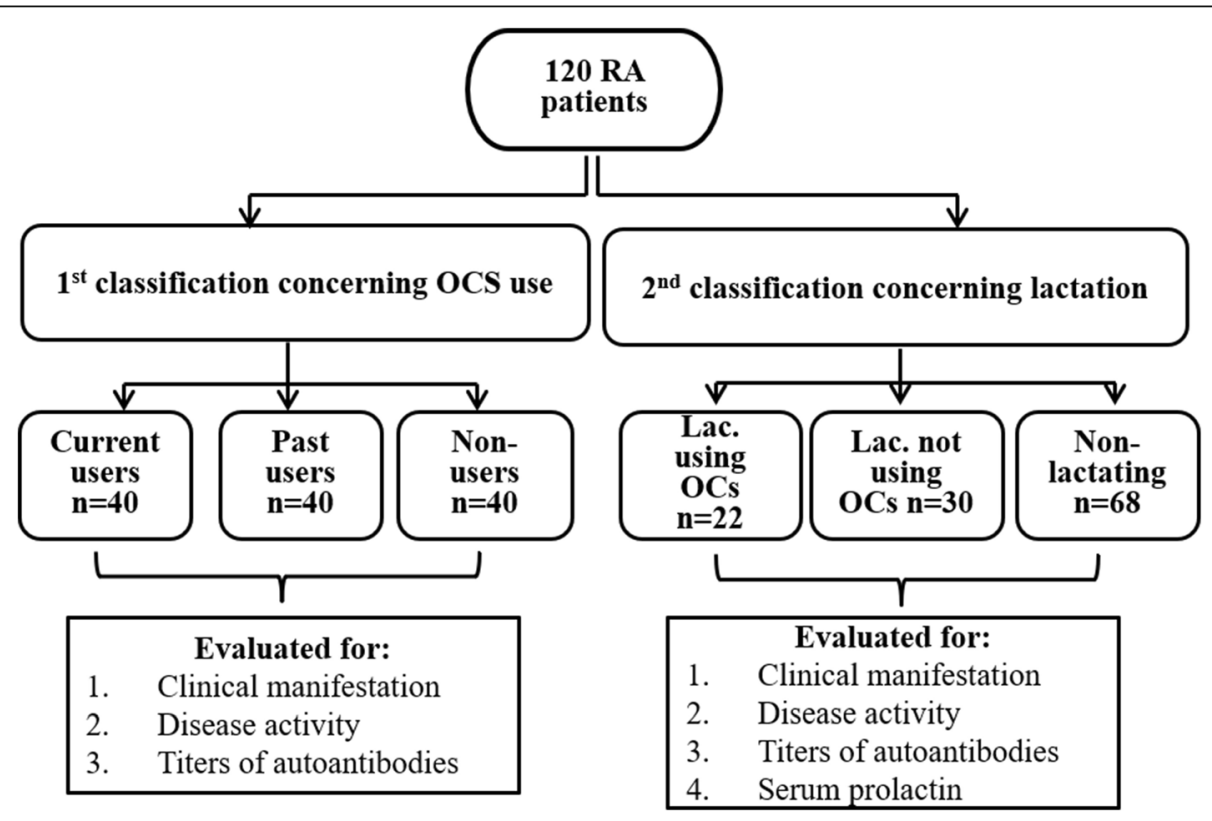

Fig. 1 Flow diagram for patients' classification and evaluation 
Table 1 Distribution of the patients according to lines of treatment and DAS28 score

\begin{tabular}{lll}
\hline Treatment & Frequency, no. (\%) & DAS28 \\
\hline MTX & $19(15.83 \%)$ & $4.9 \pm 2.3$ \\
MTX + hydroxychloroquine & $16(13.33 \%)$ & $5.15 \pm 1.75$ \\
MTX + sulphasalazine + & $8(6.66 \%)$ & $4.75 \pm 2.1$ \\
hydroxychloroquine & & \\
Leflunomide & $13(10.83 \%)$ & $6.3 \pm 1.9$ \\
MTX + leflunomide & $12(10 \%)$ & $5.3 \pm 2.7$ \\
Corticosteroids + hydroxychloroquine & $35(29.19 \%)$ & $4.6 \pm 2.4$ \\
Corticosteroids + sulphasalazine & $17(14.16 \%)$ & $5.3 \pm 1.8$ \\
\hline
\end{tabular}

\section{Effect of using oral contraceptives on disease activity,} ESR, RF, and ACPA

As shown in Fig. 2a, the DAS28 score reduced significantly $(p<0.05)$ in women currently using OCs in comparison to those who previously used OCs, the same reduction was found in DAS28 in women who previously used OCs in comparison to those who never used OCs. The DAS28 was more significantly lower $(p<$ 0.001) in current users of OCs in comparison to those who never used OCs. Using OCs did not have any significant effect on the ESR as shown in Fig. 2b. Also, using oral contraceptives did not have any significant effect on ACPA and RF titers as on comparing the ACPA and RF titers between the three groups the $p$ value was $>0.05$.

\section{Effect of using oral contraceptives during lactation on disease activity score and ESR}

As shown in Fig. 3a, lactating women not using OCs has a significantly high DAS28 $(p<0.05)$ in comparison to those women who are lactating using OCs and nonlactating. Meanwhile, there was a non-significant

Table 2 Clinical manifestations of current, past, and non-oral contraceptives users

\begin{tabular}{lll}
\hline & Mean rank & $\boldsymbol{P}$ value \\
\hline Morning stiffness & & \\
$\quad$ Current users vs past users & 35.00 vs 46.00 & $p<\mathbf{0 . 0 5}$ \\
$\begin{array}{l}\text { Past users vs non-users } \\
\text { Current users vs non-users }\end{array}$ & 33.00 vs 45.50 & $p<\mathbf{0 . 0 5}$ \\
Arthritis & 30.00 vs 51.00 & $p<\mathbf{0 . 0 0 1}$ \\
$\quad$ Current users vs past users & 36.50 vs 44.50 & $p=0.072$ \\
$\begin{array}{l}\text { Past users vs non-users } \\
\text { Current users vs non-users }\end{array}$ & 35.50 vs 44.50 & $p<\mathbf{0 . 0 5}$ \\
Extra-articular manifestations & 32.50 vs 48.50 & $p<\mathbf{0 . 0 0 1}$ \\
$\quad$ & & \\
Current users vs past users & 40.00 vs 41.00 & $p=0.823$ \\
Cast users vs non-users & 39.00 vs 42.00 & $p=0.504$ \\
\hline
\end{tabular}

difference in the DAS28 score on comparing lactating women using OCs with those non-lactating. As regards to the effect of using OCs on the ESR, there was a significant difference $(p<0.05)$ between lactating women not using OCs in comparison to lactating using OCs and non-lactating. However, there was no significant difference in the ESR between women who are lactating using OCs and women who are non-lactating (Fig. 3b). As shown in Fig. 4, there was no significant difference between the effect of using different lines of treatment on the DAS28 score within the same group, (lact + OCs + $\mathrm{C}+\mathrm{H}$ vs lact $+\mathrm{OCs}+\mathrm{C}+\mathrm{S}, p>0.05$, (lact $+\mathrm{C}+\mathrm{H}$ vs lact $+\mathrm{C}+\mathrm{S}, p>0.05)$. However, there was a significant difference on comparing lactating women using OCs with lactating not using OCs in spite of using the same lines of treatment (lact $+\mathrm{OCs}+\mathrm{C}+\mathrm{H}$ vs lact $+\mathrm{C}+\mathrm{H}$, $p<0.05$, (lact + OCs $\mathrm{C}+\mathrm{S}$ vs lact $+\mathrm{C}+\mathrm{S}, p<0.05)$.

\section{Effect of using oral contraceptives during lactation on serum prolactin, ACPA, and RF titers}

Using OCs during lactation reduced the prolactin level significantly $(p<0.05)$ in comparison to those women who are lactating not using OCs as shown in Fig. 5a, lactating women not using OCs has a significantly high serum prolactin level $(\mathrm{p}<0.05)$ and $(p<0.001)$ in comparison to those women who are lactating using OCs and non-lactating respectively. Moreover, the level of prolactin significantly $(r=0.771, p<0.001$ and $r=$ $0.831, p<0.001)$ correlated with DAS28 score in lactating using OCs, and lactating not using OCs respectively as shown in Fig. 5b, c. On the other hand, using oral contraceptives during lactation did not have any significant effect on ACPA and RF titers as on comparing the ACPA and RF titers between the three groups the $p$ value was $>0.05$.

Table 3 Clinical manifestations of lactating women using OCs, lactating not using OCs and non-lactating

\begin{tabular}{lll}
\hline & Mean Rank & $P$ value \\
\hline Morning stiffness & & \\
Lactating using OCs vs lactating not using & 24.18 vs 28.20 & $p=0.257$ \\
Lactating using OCs vs non-lactating & 36.55 vs 48.40 & $p<0.05$ \\
Lactating not using vs non-lactating & 45.80 vs 51.13 & 0.236 \\
Arthritis & & \\
Lactating using OCs vs lactating not using & 19.45 vs 31.67 & $p<0.001$ \\
Lactating using OCs vs non-lactating & 33.36 vs 49.43 & $p<0.01$ \\
Lactating not using vs non-lactating & 53.33 vs 47.81 & $p=0.234$ \\
Extra-articular manifestations & & \\
Lactating using OCs vs lactating not using & 24.27 vs 28.13 & $p=0.286$ \\
Lactating using OCs vs non-lactating & 39.32 vs 47.50 & $p=0.139$ \\
Lactating not using vs non-lactating & 48.37 vs 50.00 & $p=0.762$ \\
\hline
\end{tabular}



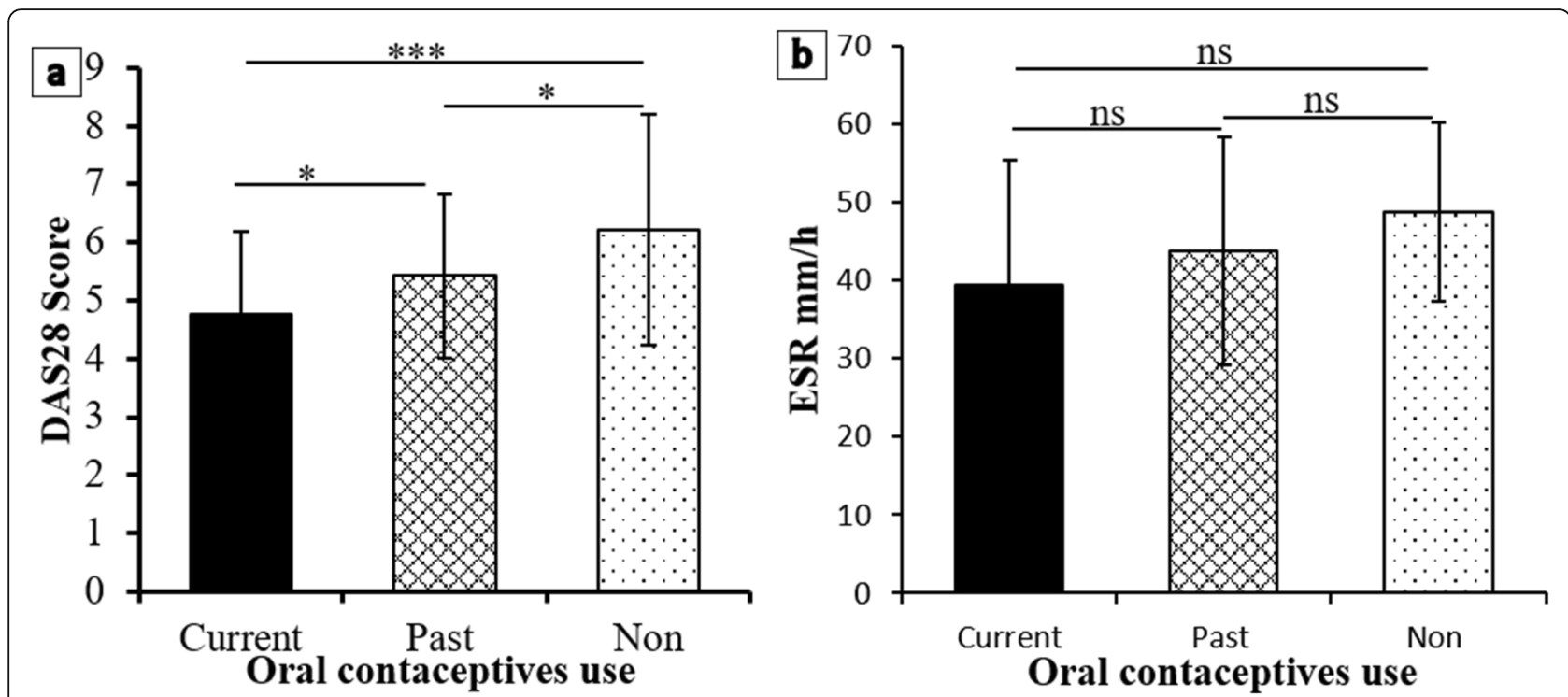

Fig. 2 Effect of oral contraceptives on DAS28 score and ESR. a DAS28 score. b ESR. $n=40$ for each group, * significant difference at $p<0.05$, ***very high significant difference at $p<0.001, \mathrm{~ns}=$ non-significant difference

\section{Discussion}

The etiology and pathogenesis of RA are still unknown; however, sex hormones may have a role in modulating disease onset and progression [14]. Females are more commonly affected than males. Progesterone and androgens have suppressive effects over the immune system, while prolactin has a stimulatory effect, and estrogen can have either effect [25]. The disease activity of RA patients improves during pregnancy due to some changes in the maternal immune system, occurring through multiple immunological mechanisms that stop after delivery leading to increased postpartum disease activity [26]. The increase in estrogen and progesterone levels during pregnancy was found to have a protective effect against both the development and activity of RA $[27,28]$. It is reported that combined oral contraceptives may have a tendency of controlling disease activity in RA patients [29].
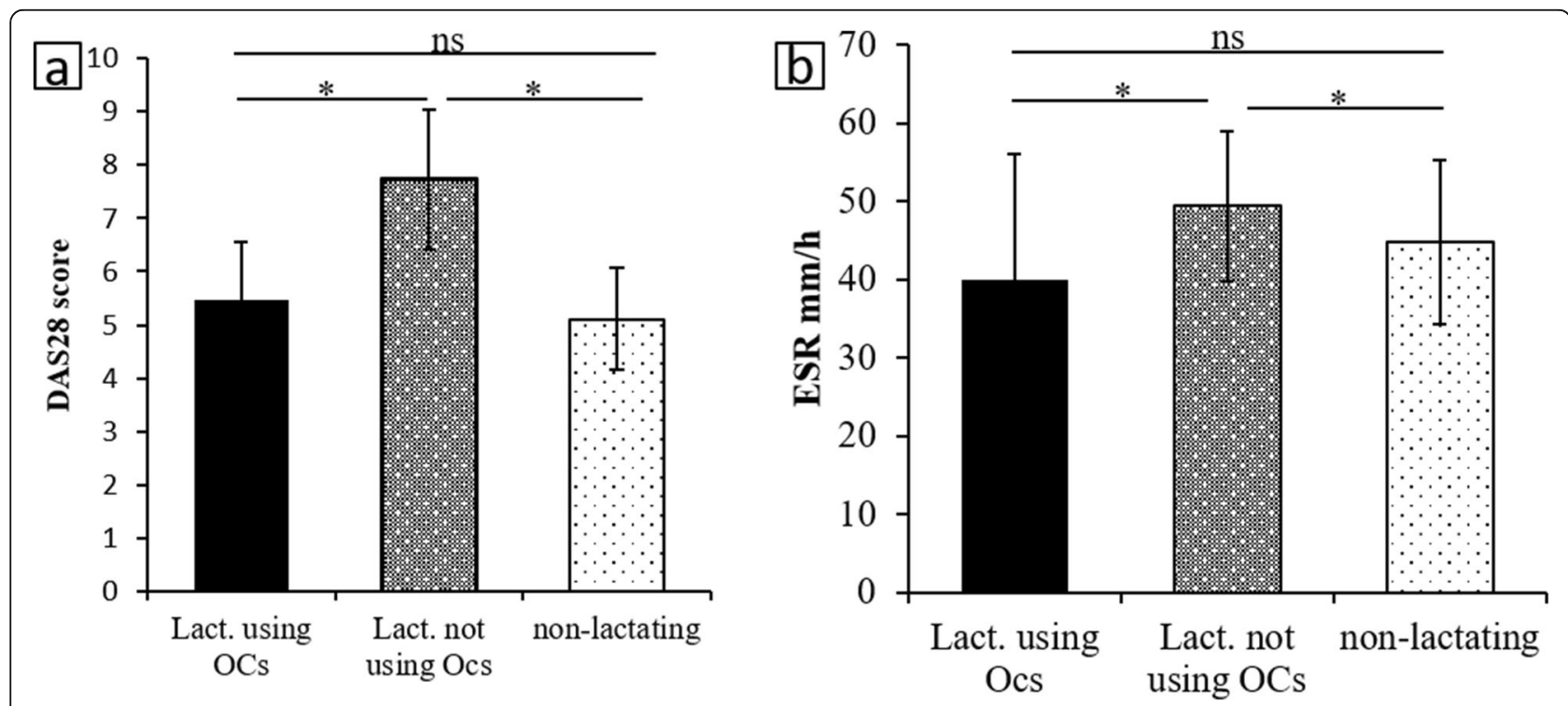

Fig. 3 Effect of using oral contraceptives during lactation on DAS28 score and ESR. a DAS28 score. $\mathbf{b}$ ESR, data presented as mean \pm SD, ns $=$ insignificant difference, ${ }^{*}$ significant difference at $p<0.05$ 


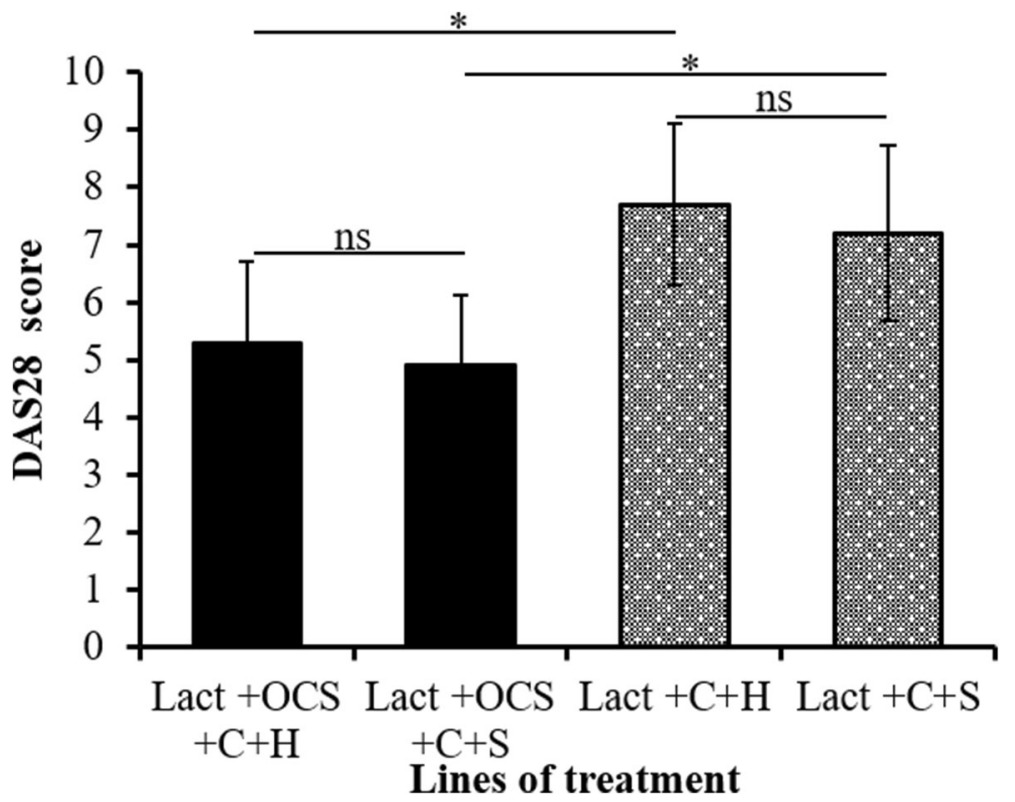

Fig. 4 Effect of different lines of treatment in lactating women on DAS28 score. Where Lac $+\mathrm{OCs}+\mathrm{C}+\mathrm{H}$, lactating women using OCs and treated by corticosteroids + hydroxychloroquine $n=16$. Lac + OCs $+C+S$, lactating women using OCs and treated by corticosteroids + sulphasalazine $n=6$. LaC $+\mathrm{C}+\mathrm{H}$, lactating women not using OCs and treated by corticosteroids + hydroxychloroquine $n=19$. Lac $+\mathrm{C}+\mathrm{S}$, lactating women not using OCs and treated by corticosteroids + sulphasalazine, $n=11$. Where ${ }^{*}$ is significant difference at $p<0.05$ and ns, non-significant difference

Our findings revealed that using oral contraceptives significantly reduced the clinical manifestations. Women who are currently using and previously used OCs presented with less morning stiffness and arthritis. The effect of OCs was more pronounced on the DAS28 score; current users of OCs had significantly low DAS28 scores in comparison to past users and both of them had a lower score when compared with non-users. Our findings agree with Amini et al. who found that the activity and severity of rheumatoid arthritis can be improved by oral contraceptive pills [30]. Also, Brouwer et al. reported that oral contraceptives are considered the best option for RA patients as they positively affect the disease activity [31]. Fayed et al. found that RA patients who are using combined oral contraceptives have the lowest disease severity in comparison to the other methods of contraception [32]. However, using OCs did not have any significant effect on ESR; this agrees with Amini et al. [30], and it also had no significant effect on ACPA and RF titers in all studied groups.

The combined OCs contain two anti-inflammatory agents; estrogen and progesterone. The potential protective effect of OCs on RA may be explained by more than one mechanism; the first, estrogen in OCs can suppress the immune response and downregulate proinflammatory cytokines production; the second, estrogen may prevent the formation of osteoclasts and pannus formation, hence decreasing RA progression [33]. Moreover, progesterone prevents the production of immunoglobulins and pro-inflammatory cytokines [34].

Using OCs during lactation reduced the morning stiffness in lactating women using OCs in comparison to those non-lactating. However, its effect on arthritis was more pronounced where arthritis was significantly reduced on using OCs. Concerning disease activity, lactating women who are not using OCs had a significantly high DAS28 in comparison to those women who are lactating using OCs and non-lactating. Despite the wellknown role of therapy in controlling disease activity in RA patients, we suppose that the reduction in the disease activity in lactating women using OCs was more related to the use of OCs than the effect of therapy, this may be supported by the finding that the same two lines of treatment were used in the two groups but OCs using group had lower DAS28 in comparison to OCs nonusers. Regarding serum prolactin, lactating women not using OCs have significantly high serum prolactin in comparison to lactating using OCs and non-lactating. Moreover, the prolactin levels significantly correlated with DAS28 in all groups. This may be explained by the role of increased serum prolactin in enhancing postpartum disease flare in RA patients. Meanwhile, there was a non-significant difference in the DAS28 score on comparing lactating women using OCs with those non- 

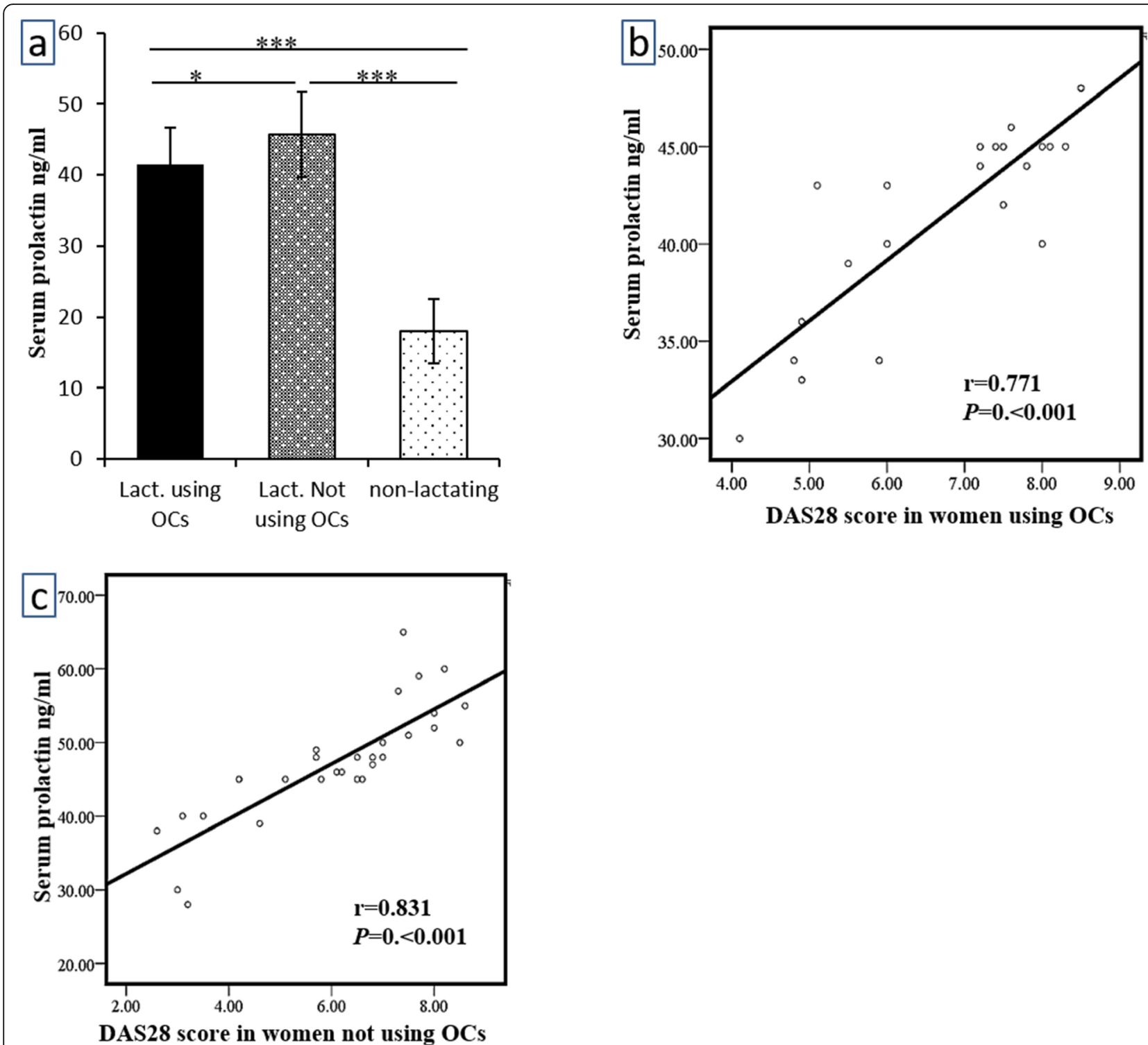

Fig. 5 Effect of oral contraceptives on serum prolactin and its correlation with disease activity. a Serum prolactin level. b Correlation between serum prolactin and DAS28 in lactating women using oral contraceptives. c Correlation between serum prolactin and DAS28 in lactating women not using oral contraceptives. Pearson's correlation was used for calculation of the correlation $r=$ correlation coefficient, ${ }^{*}$ significant difference at $p<0.05{ }^{* *}$ high significant difference at $p<0.01$, and ${ }^{* * *}$ very high significant difference at $p<0.001$

lactating. Our results agree with Fayez et al. who reported that increased level of serum prolactin was found in patients with high disease activity when compared to moderate and low disease activity and serum prolactin level was significantly correlated with DAS28 and ESR [20], also, Fojtíková et al. reported that RA patients with active disease had high serum prolactin level which correlated significantly with disease activity and Larsen score [35].

Many previous studies have explored the effect of breastfeeding on disease activity during the postpartum period which is challenging for women suffering from rheumatologic diseases due to the increased risk of disease flare and limitations in the use of medications during breastfeeding [36]. Barrett et al. found that breastfeeding women with inflammatory polyarthritis developed a more aggressive disease 6 months later in comparison to previous breastfeeders and nonbreastfeeders [16]. The disease activity of the majority of RA patients increases during the first 3 months after delivery, and in few patients, it increases by 9 months; thus, women with RA might benefit from being nonbreastfeeders [15]. The prolactin release during breastfeeding could also play a role in the disease flare in the 
postpartum period [19]. Our results concerning disease activity during lactation agree with the previously mentioned studies; but interestingly, we found that lactating women using OCs had low disease activity in comparison to those lactating not using OCs, and their disease activity was near to that of non-lactating women; hence, OCs may be considered the most suitable contraceptive method for lactating RA women with the additional benefit of improving disease activity. As regards to the effect of using OCs on the ESR, lactating women using OCs had a significantly low ESR in comparison to those who are not using OCs.

Hormonal changes alongside changes in cytokine patterns and immune cells are all factors contributing to the postpartum disease flare in RA patients [37]. Another theory that may contribute to the postpartum RA disease flare is the prolactin hormone which increases during breastfeeding $[15,38]$. Prolactin is considered to have a dual action in the joint tissues, while it prevents the apoptosis of chondrocytes by activating antiapoptotic genes, activates the proliferation of synovial cells, and enhances the synthesis of IL-6, IL-8, and matrix metalloproteinase, so it is suggested that high prolactin either produced locally in joints by chondrocytes or obtained from the circulating blood may have a role in structural changes leading to the development of RA [39].

Prolactin level is normally high in lactating women but the effect of using OCs on the serum prolactin level is still controversial. Our results indicated that using OCs significantly reduced serum prolactin. Our finding agrees with Ryu et al. who found that the use of combined OCs decreased the serum prolactin level in lactating women [40], also Clendenen et al. found that parity and OCs use was associated with a decreased level of serum prolactin and ovarian cancer risk [41]. Testa et al. reported that using combined OCs insignificantly decreased serum prolactin [42]. On the other hand, our results are in contradicting with Alvarez-Tutor et al. who reported that serum prolactin significantly increased on using combined OCs [43]. There are controversies whether the content of estrogen in OCs affects serum prolactin or not [44]; Josimovich et al. found that high estrogen OCs increases serum prolactin within the first 6 months of its use but this effect is not manifested after 6 months, while low estrogen OCs either decrease serum prolactin or have no effect depending on basal prolactin value [45].

Prolactin has a strong immunomodulatory effect through stimulating the transcription of the gene of interferon regulatory factor IRF-1, and modulation of the expression of IL-12, and other pro-inflammatory cytokines like tumor necrosis factor TNF- $\alpha$, and interferon (IFN)- $\alpha$, it also increases the survival of both B cells and autoreactive $\mathrm{T}$ cells through its anti-apoptotic activities [35]. Prolactin stimulates the immune system mainly through the inhibition of the autoreactive B lymphocytes' negative selection [15]. Our findings reinforce the concept that the levels of estrogen, progesterone, and prolactin change after delivery affecting the cytokines and immune cells and this in turn modulates the RA disease activity. Meanwhile, using OCs during lactation may have a tendency of controlling disease activity in RA patients, as lactating women not using OCs had the highest prolactin level and DAS28 score and those using OCs had significantly reduced serum prolactin and DAS28 score.

\section{Conclusion}

Oral contraceptives may be considered in RA female patients not only as a suitable birth control method but also it has a controlling effect on disease activity even during lactation

\section{Study limitations}

Further studies with a big sample size are required comparing the effect of progesterone only and combined oral contraceptive pills on disease activity in RA patients.

\section{Abbreviations}

MTX: Methotrexate; RA: Rheumatoid arthritis; OCs: Oral contraceptives: DAS28: Disease activity score-28; ESR: Erythrocyte sedimentation rate; RF: Rheumatoid factor; ACPA: Anti-citrullinated protein antibodies; ACR: American College of Rheumatology

\section{Acknowledgements}

Not applicable.

\section{Submission declaration and verification \\ This manuscript has not been published previously and it is not under consideration for publication elsewhere.}

\section{Authors' contributions}

The conceptualization of this study, selection and diagnosis of patients were performed by S.A.E., and collection of data was performed by D.M.H. The manuscript was written and the data interpretation was performed by S.A. E. The manuscript was revised by E.M.A. All authors have read and approved the manuscript

\section{Funding}

This study had no funding from any resource.

\section{Availability of data and materials \\ The data of the current study are available from the corresponding author on reasonable request.}

\section{Ethics approval and consent to participate}

This study was carried out in accordance with the ethical standards laid down in the Helsinki Declaration of 1975 and its later amendments in 2000, and approved by the Medical Research Ethics Committee, Faculty of Medicine, Sohag University, under the reference number IBR\#S20-149. All patients included in this study gave written informed consent to participate in this research.

\section{Consent for publication}

All patients included in this research gave written informed consent to publish the data contained within this study. 


\section{Competing interests}

None

Received: 17 September 2020 Accepted: 29 October 2020 Published online: 13 November 2020

\section{References}

1. Majithia V, Geraci SA (2007) Rheumatoid arthritis: diagnosis and management. Am J Med 120:936-939

2. Smolen JS, Aletaha D, McInnes IB (2016) Rheumatoid arthritis. Lancet 388 : 2023-2038

3. Oliver JE, Silman AJ (2009) Why are women predisposed to autoimmune rheumatic diseases? Arthritis Res Ther 11:252

4. Tedeschi SK, Bermas B, Costenbader KH (2013) Sexual disparities in the incidence and course of SLE and RA. Clin Immunol 149:211-218

5. Sokka T, Toloza S, Cutolo M, Kautiainen H, Makinen H, Gogus F, Skakic V, Badsha H, Peets T, Baranauskaite A et al (2009) Women, men, and rheumatoid arthritis: analyses of disease activity, disease characteristics, and treatments in the QUEST-RA study. Arthritis Res Ther 11:R7

6. Sparks J, Chang S-C, Liao K, Lu B, Solomon D, Costenbader K, Karlson E (2014) Incident rheumatoid arthritis and risk of mortality among women followed prospectively from 1976 to 2010 in the nurses' health study: 818 . Arthritis Rheumatol 66

7. Kuller LH, Mackey RH, Walitt BT, Deane KD, Holers VM, Robinson WH, Sokolove J, Chang Y, Liu S, Parks CG et al (2014) Determinants of mortality among postmenopausal women in the women's health initiative who report rheumatoid arthritis. Arthritis Rheumatol (Hoboken, N.J.) 66:497-507

8. Simon TA, Thompson A, Gandhi KK, Hochberg MC, Suissa S (2015) Incidence of malignancy in adult patients with rheumatoid arthritis: a meta-analysis. Arthritis Res Ther 17:212

9. Alpízar-Rodríguez D, Pluchino N, Canny G, Gabay C, Finckh A (2017) The role of female hormonal factors in the development of rheumatoid arthritis. Rheumatology (Oxford, England) 56:1254-1263

10. Berglin E, Kokkonen H, Einarsdottir E, Agren A, Rantapää Dahlqvist S (2010) Influence of female hormonal factors, in relation to autoantibodies and genetic markers, on the development of rheumatoid arthritis in northern Sweden: a case-control study. Scand J Rheumatol 39:454-460

11. Harlow SD, Gass M, Hall JE, Lobo R, Maki P, Rebar RW, Sherman S, Sluss PM, de Villiers TJ (2012) Executive summary of the Stages of Reproductive Aging Workshop + 10: addressing the unfinished agenda of staging reproductive aging. J Clin Endocrinol Metab 97:1159-1168

12. Scott IC, Steer S, Lewis CM, Cope AP (2011) Precipitating and perpetuating factors of rheumatoid arthritis immunopathology: linking the triad of genetic predisposition, environmental risk factors and autoimmunity to disease pathogenesis. Best Pract Res Clin Rheumatol 25:447-468

13. Hazes JM, Coulie PG, Geenen V, Vermeire S, Carbonnel F, Louis E, Masson P, De Keyser F (2011) Rheumatoid arthritis and pregnancy: evolution of disease activity and pathophysiological considerations for drug use. Rheumatology (Oxford, England) 50:1955-1968

14. Cutolo M, Straub RH (2009) Insights into endocrine-immunological disturbances in autoimmunity and their impact on treatment. Arthritis Res Ther 11:218

15. Vieira Borba $V$, Shoenfeld $Y$ (2019) Prolactin, autoimmunity, and motherhood: when should women avoid breastfeeding? Clin Rheumatol 38: 1263-1270

16. Barrett JH, Brennan P, Fiddler M, Silman A (2000) Breast-feeding and postpartum relapse in women with rheumatoid and inflammatory arthritis. Arthritis Rheum 43:1010-1015

17. Carreño PC, Sacedón R, Jiménez E, Vicente A, Zapata AG (2005) Prolactin affects both survival and differentiation of T-cell progenitors. J Neuroimmunol 160:135-145

18. Saha S, Gonzalez J, Rosenfeld G, Keiser H, Peeva E (2009) Prolactin alters the mechanisms of B cell tolerance induction. Arthritis Rheum 60:1743-1752

19. Tang MW, Garcia S, Gerlag DM, Tak PP, Reedquist KA (2017) Insight into the endocrine system and the immune system: a review of the inflammatory role of prolactin in rheumatoid arthritis and psoriatic arthritis. Front Immunol 8:720

20. Fayez D, Habeeb R, Hassan S, Abdelfatah N (2015) Serum prolactin in patients with rheumatoid arthritis and its association with disease activity. Egypt J Rheumatol Clin Immunol 3:55-60
21. Shelly S, Boaz M, Orbach H (2012) Prolactin and autoimmunity. Autoimmun Rev 11:A465-A470

22. Camacho EM, Lunt M, Farragher TM, Verstappen SM, Bunn DK, Symmons DP (2011) The relationship between oral contraceptive use and functional outcome in women with recent-onset inflammatory polyarthritis: results from the Norfolk Arthritis Register. Arthritis Rheum 63:2183-2191

23. Chen Q, Jin Z, Xiang C, Cai Q, Shi W, He J (2014) Absence of protective effect of oral contraceptive use on the development of rheumatoid arthritis: a meta-analysis of observational studies. Int J Rheum Dis 17:725-737

24. Qian Q, Liuqin L, Hao L, Shiwen Y, Zhongping Z, Dongying C, Fan L, Hanshi $X$, Xiuyan Y, Yujin Y (2015) The effects of bromocriptine on preventing postpartum flare in systemic lupus erythematosus patients from South China. J Immunol Res 2015:316965

25. Van Vollenhoven RF, McGuire JL (1994) Estrogen, progesterone, and testosterone: can they be used to treat autoimmune diseases? Cleve Clin J Med 61:276-284

26. Förger F, Villiger PM (2020) Immunological adaptations in pregnancy that modulate rheumatoid arthritis disease activity. Nat Rev Rheumatol 16:113122

27. Straub RH (2007) The complex role of estrogens in inflammation. Endocr Rev 28:521-574

28. Szekeres-Bartho J, Barakonyi A, Par G, Polgar B, Palkovics T, Szereday L (2001) Progesterone as an immunomodulatory molecule. Int Immunopharmacol 1:1037-1048

29. Benagiano G, Benagiano M, Bianchi P, D'Elios MM, Brosens I (2019) Contraception in autoimmune diseases. Best Pract Res Clin Obstet Gynaecol 60:111-123

30. Amini L, Kalhor M, Haghighi A, Seyedfatemi N, Hosseini F (2018) Effect of oral contraceptive pills on rheumatoid arthritis disease activity in women: a randomized clinical trial. Med J Islam Repub Iran 32:61

31. Brouwer J, Laven JS, Hazes JM, Dolhain RJ (2015) Brief report: miscarriages in female rheumatoid arthritis patients: associations with serologic findings, disease activity, and antirheumatic drug treatment. Arthritis Rheumatol (Hoboken, N.J.) 67:1738-1743

32. Fayed HL, Hatem H, Kamal HA, Mahmoud I (2017) Rheumatoid arthritis activity and severity in relation to commonly used contraceptive methods in a cohort of Egyptian female patients. Int J Clin Rheumatol 12:168

33. Pullerits R, d'Elia HF, Tarkowski A, Carlsten H (2009) The decrease of soluble RAGE levels in rheumatoid arthritis patients following hormone replacement therapy is associated with increased bone mineral density and diminished bone/cartilage turnover: a randomized controlled trial. Rheumatology (Oxford, England) 48:785-790

34. Trigunaite A, Dimo J, Jørgensen TN (2015) Suppressive effects of androgens on the immune system. Cell Immunol 294:87-94

35. Fojtíková M, Tomasová Studýnková J, Filková M, Lacinová Z, Gatterová J, Pavelka K, Vencovský J, Senolt L (2010) Elevated prolactin levels in patients with rheumatoid arthritis: association with disease activity and structural damage. Clin Exp Rheumatol 28:849-854

36. Bermas BL (2017) Lactation and management of postpartum disease. Rheum Dis Clin North Am 43:249-262

37. Østensen M, Sicher P, Förger F, Villiger PM (2005) Activation markers of peripheral blood mononuclear cells in late pregnancy and after delivery: a pilot study. Ann Rheum Dis 64:318-320

38. Recalde G, Moreno-Sosa T, Yúdica F, Quintero CA, Sánchez MB, Jahn GA, Kalergis AM, Mackern-Oberti JP (2018) Contribution of sex steroids and prolactin to the modulation of $\mathrm{T}$ and $\mathrm{B}$ cells during autoimmunity. Autoimmun Rev 17:504-512

39. Zermeño C, Guzmán-Morales J, Macotela Y, Nava G, López-Barrera F, Kouri JB, Lavalle C, de la Escalera GM, Clapp C (2006) Prolactin inhibits the apoptosis of chondrocytes induced by serum starvation. J Endocrinol 189: $R 1-R 8$

40. Ryu K, Yu HK, Kwak HM, Hong SS (1983) Effects of combined oral contraceptive steroids on pituitary-ovarian function during the menstrual cycle of lactating women. Contraception 27:299-310

41. Clendenen TV, Arslan AA, Lokshin AE, Liu M, Lundin E, Koenig KL, Berrino F, Hallmans G, Idahl A, Krogh V et al (2013) Circulating prolactin levels and risk of epithelial ovarian cancer. Cancer Causes Control 24:741-748

42. Testa G, Vegetti W, Motta T, Alagna F, Bianchedi D, Carlucci C, Bianchi M, Parazzini F, Crosignani PG (1998) Two-year treatment with oral contraceptives in hyperprolactinemic patients. Contraception 58:69-73 
43. Alvarez-Tutor E, Forga-LLenas L, Rodriguez-Erdozain R, Goñi-Iriarte MJ, Menendez-Torre E, Alvarez-Tutor J (1999) Persistent increase of PRL after oral contraceptive treatment. Arch Gynecol Obstet 263:45-50

44. Molitch ME (2008) Drugs and prolactin. Pituitary 11:209-218

45. Josimovich JB, Lavenhar MA, Devanesan MM, Sesta HJ, Wilchins SA, Smith AC (1987) Heterogeneous distribution of serum prolactin values in apparently healthy young women, and the effects of oral contraceptive medication. Fertil Steril 47:785-791

\section{Publisher's Note}

Springer Nature remains neutral with regard to jurisdictional claims in published maps and institutional affiliations.

\section{Submit your manuscript to a SpringerOpen ${ }^{\circ}$ journal and benefit from:}

- Convenient online submission

- Rigorous peer review

- Open access: articles freely available online

- High visibility within the field

- Retaining the copyright to your article

Submit your next manuscript at $\boldsymbol{\nabla}$ springeropen.com 